\title{
CAYLEY'S THEOREM AND ADAMS COMPLETION
}

\author{
SNIGDHA BHARATI CHOUDHURY AND A. BEHERA
}

Received 20 January, 2015

\begin{abstract}
Cayley's Theorem states that a permutation group of a group is isomorphic to the given group. We show that this permutation group is Adams completion of the group with respect to a suitably chosen set of morphisms in the category of groups and homomorphisms.
\end{abstract}

2010 Mathematics Subject Classification: 18B40; 55P60

Keywords: category of fractions, calculus of left fractions, Grothendieck universe, Adams completion

\section{INTRODUCTION}

Many algebraic and geometrical constructions in algebraic and general topology can be viewed as Adams completions or cocompletions of objects in suitable categories, with respect to the chosen set of morphisms. The notion of generalized Adams completion arose from a general categorical completion process, suggested by Adams [1,2]. Originally, this was considered for admissible categories and generalized homology (or cohomology) theories. The notion has been considered in a more general framework by Deleanu, Frei and Hilton [4] where an arbitrary category and an arbitrary set of morphisms of the category are considered.

Let $\mathscr{C}$ be a category and $S$ be a set of morphisms of $\mathscr{C}$. Let $\mathscr{C}\left[S^{-1}\right]$ denote the category of fractions [6] of $\mathscr{C}$ with respect to $S$ and $F: \mathscr{C} \rightarrow \mathscr{C}\left[S^{-1}\right]$ be the canonical functor. Let $\mathscr{S}$ denote the category of sets and functions. Then for a given object $Y$ of $\mathscr{C}, \mathscr{C}\left[S^{-1}\right](-, Y): \mathscr{C} \rightarrow \mathscr{S}$ defines a contravariant functor [6]. If this functor is representable by an object $Y_{S}$ of $\mathscr{C}$, i.e., $\mathscr{C}\left[S^{-1}\right](-, Y) \cong \mathscr{C}\left(-, Y_{S}\right)$, then $Y_{S}$ is called the generalized Adams completion [4] of $Y$ with respect to the set of morphisms $S$ or simply the $S$-completion of $Y . Y_{S}$ is also referred as the completion of $Y$.

Let $\bar{S}$ be the set of all morphisms $s$ in $\mathscr{C}$ such that $F(s)$ is an isomorphism in $\mathscr{C}\left[S^{-1}\right]$. Then $\bar{S}$ is called as the saturation of $S$. The set of morphisms $S$ is said to be saturated [4] if $S=\bar{S}$. 


\section{CAyley's Theorem}

Let $G$ be a group. Then $\bar{G}=\left\{T_{g}: G \rightarrow G \mid T_{g}(x)=g x\right.$ for all $\left.x \in G, g \in G\right\}$ is a permutation group. Then by Cayley's Theorem [5] $G$ is isomorphic to $\bar{G}$. The following result is easy to prove.

Theorem 1. Let $G$ be a group and $\bar{G}$ be a group of permutations. Then there is an isomorphism $\varphi: G \rightarrow \bar{G}$. If $K$ is a group and $f: G \rightarrow K$ is an isomorphism, then there exists a unique isomorphism $\theta: K \rightarrow \bar{G}$ making the triangle commutative, i.e., $\theta f=\varphi$.



Proof. Define $\theta: K \rightarrow \bar{G}$ by the rule $\theta(k)=\varphi f^{-1}(k)$ for all $k \in K$. Clearly, $\theta$ is well defined and is also a homomorphism. In order to show $\theta$ is injective, we have to show $\operatorname{ker} \theta=\left\{e_{K}\right\}$. Let $k \in \operatorname{ker} \theta$, i.e., $\theta(k)=\varphi f^{-1}(k)=e_{\bar{G}}$. So $f^{-1}(k)=e_{G}$, i.e., $k=e_{K}$, showing $\theta$ is injective. Next we show $\theta$ is surjective. $\theta(K)=\varphi f^{-1}(K)=$ $\varphi(G)=\bar{G}$; so $\theta$ is surjective. Thus, $\theta$ is an isomorphism.

For any $g \in G, \theta f(g)=\varphi f^{-1}(f(g))=\varphi(g)$. Thus $\theta f=\varphi$, i.e., the diagram is commutative. Next we show that $\theta$ is unique. Let there exist another $\theta^{\prime}: K \rightarrow \bar{G}$ with $\theta^{\prime} f=\varphi$. Then for any $k \in K, \theta(k)=\varphi f^{-1}(k)=\theta^{\prime} f f^{-1}(k)=\theta^{\prime}(k)$. Hence $\theta=\theta^{\prime}$. This completes the proof of Theorem 1 .

\section{THE CATEGORY $\mathscr{G}$}

Let $\mathscr{U}$ be a fixed Grothendieck universe [6]. Let $\mathscr{G}$ denote the category of groups and homomorphisms. We assume that the underlying sets of the elements of $\mathscr{G}$ are elements of $\mathscr{U}$.

Let $S$ be the set of all morphisms $s: P \rightarrow Q$ in $\mathscr{G}$ such that $s$ is an isomorphism.

Theorem 2. Let $s_{i}: P_{i} \rightarrow Q_{i}$ lie in $S$ for each $i \in I$ where the index set $I$ is an element of $\mathscr{U}$. Then $\underset{i \in I}{\vee} s_{i}: \underset{i \in I}{\vee} P_{i} \rightarrow \underset{i \in I}{\vee} Q_{i}$ lies in $S$.

Proof. Coproducts in $\mathscr{G}$ are the free products. Define a map $s=\underset{i \in I}{\vee} s_{i}: P=$ $\underset{i \in I}{\vee} P_{i} \rightarrow \underset{i \in I}{\vee} Q_{i}=Q$ by the rule $s\left(p_{1} \cdots p_{k}\right)=\varphi\left(p_{1}\right) \cdots \varphi\left(p_{k}\right)$ where $\varphi\left(p_{j}\right)=s_{i}\left(p_{j}\right)$ if $p_{j} \in P_{i}, j=1, \cdots, k$. Clearly, $s$ is well defined and is also a homomorphism.

In order to show $s$ is injective we have to show ker $s=\left\{e_{P}\right\}$. Let $p=p_{1} \cdots p_{k} \in$ ker $s$, i.e., $s\left(p_{1} \cdots p_{k}\right)=e_{Q}=1$; this implies that $\varphi\left(p_{1}\right) \cdots \varphi\left(p_{k}\right)=1$ where $\varphi\left(p_{j}\right)=$ $s_{i}\left(p_{j}\right)=\omega_{i}^{\prime}\left(s_{i}\left(p_{j}\right)\right)$ for $p_{j} \in P_{i}, j=1, \cdots, k$ and $\omega_{i}^{\prime}: Q_{i} \rightarrow Q$ defined by $\omega_{i}^{\prime}\left(e_{Q_{i}}\right)=$ 1 and $\omega_{i}^{\prime}(b)=b$ for $b \in Q_{i}$, is a monomorphism for each $i \in I$. So each $\varphi\left(p_{j}\right)=$ $s_{i}\left(p_{j}\right)=\omega_{i}^{\prime}\left(s_{i}\left(p_{j}\right)\right)=1=\omega_{i}^{\prime}\left(e_{Q_{i}}\right)$ from which it follows that $s_{i}\left(p_{j}\right)=e_{Q_{i}}$, i.e., 
$p_{j}=e_{P_{i}}$ for $p_{j} \in P_{i}$ and $j=1, \cdots, k$. Next $p_{1} \cdots p_{k}=\eta\left(p_{1}\right) \cdots \eta\left(p_{k}\right)$ where $\eta\left(p_{j}\right)=\omega_{i}\left(p_{j}\right)=\omega_{i}\left(e_{P_{i}}\right)=1$ for $p_{j} \in P_{i}$ and $\omega_{i}: P_{i} \rightarrow P$ is defined by $\omega_{i}\left(e_{P_{i}}\right)=$ 1 and $\omega_{i}(a)=a$ for $a \in P_{i}$, is a monomorphism for each $i \in I$. So $p_{1} \cdots p_{k}=1=e_{P}$. Hence $s$ is injective.

Next take $q_{1} \cdots q_{k} \in Q$ where $q_{j} \in Q_{i}$ for $i \in I$ and $j=1, \cdots, k$. But $q_{j}=s_{i}\left(p_{j}\right)$ where $p_{j} \in P_{i}$. So $q_{1} \cdots q_{k}=\varphi\left(p_{1}\right) \cdots \varphi\left(p_{k}\right)$ where $\varphi\left(p_{j}\right)=s_{i}\left(p_{j}\right)$ for $p_{j} \in P_{i}$. Hence $q_{1} \cdots q_{k}=s\left(p_{1} \cdots p_{k}\right)$, showing $s$ is surjective. Therefore, $s: P \rightarrow Q$ is an isomorphism, i.e., $s=\underset{i \in I}{\vee} s_{i}$ lies in $S$. This completes the proof of Theorem 2.

We will show that the set of morphisms $S$ of the category $\mathscr{G}$ of groups and homomorphisms admits a calculus of left fractions [6].

Theorem 3. $S$ admits a calculus of left fractions.

Proof. Since $S$ consists of all isomorphisms in $\mathscr{G}$, clearly $S$ is a closed family of morphisms of the category $\mathscr{G}$. We shall verify conditions (i) and (ii) of Theorem 1.3 ([4], p.67). Let $s: P \rightarrow Q$ and $t: Q \rightarrow R$ be two morphisms in $\mathscr{G}$. We show if $t s \in S$ and $s \in S$, then $t \in S$. Let $q \in \operatorname{ker} t$, i.e., $t(q)=e_{R}$. So $t(s(p))=e_{R}, p \in P$. Since $t s$ is an isomorphism we have $p=e_{P}$. So $q=s\left(e_{P}\right)=e_{Q}$, i.e., $\operatorname{ker} t=\left\{e_{Q}\right\}$, i.e., $t$ is injective. Since $t s \in S$ and $s \in S$, we have $t s(P)=R$ and $s(P)=Q$. Then $t(Q)=t(s(P))=R$. So $t$ is surjective. Thus $t$ is an isomorphism, i.e., $t \in S$. Hence condition (i) of Theorem 1.3 ([4], p.67) holds.

In order to prove condition (ii) of Theorem 1.3 ([4], p.67) consider the diagram

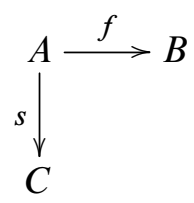

in $\mathscr{G}$ with $s \in S$. We assert that the above diagram can be completed to a weak push-out diagram



in $\mathscr{G}$ with $t \in S$. Let $D=(B * C) / N$, where $N$ is a normal subgroup of $B * C$ generated by $\left\{f(a) s(a)^{-1}: a \in A\right\}$. Define $t: B \rightarrow D$ by the rule $t(b)=b N$ for all $b \in B$ and $g: C \rightarrow D$ by the rule $g(c)=c N$ for all $c \in C$. Clearly, the two maps are well defined and homomorphisms. For any $a \in A, t f(a)=f(a) N=s(a) N=$ $g s(a)$, implies that $t f=g s$. Hence the diagram is commutative.

Next we show $t \in S$, i.e., $t$ is an isomorphism. Take $b \in \operatorname{ker} t$, i.e., $t(b)=e_{D}=N$; this implies $b N=N$, i.e., $b \in N$. Hence $b=f(a) s(a)^{-1}=f(a) s\left(a^{-1}\right)$ for some $a \in A$. Then $b 1=f(a) s\left(a^{-1}\right)$, implies that $b \delta_{2}\left(e_{C}\right)=f(a) \delta_{2}\left(s\left(a^{-1}\right)\right)$ where $\delta_{2}$ : 
$C \rightarrow B * C$, defined by $\delta_{2}\left(e_{C}\right)=1$ and $\delta_{2}(c)=c$ for $c \in C$, is a monomorphism. Then $b=f(a), \delta_{2}\left(e_{C}\right)=\delta_{2}\left(s\left(a^{-1}\right)\right)$. As $\delta_{2}\left(e_{C}\right)=\delta_{2}\left(s\left(a^{-1}\right)\right)$, we have $s\left(a^{-1}\right)=$ $e_{C}$, giving $a=e_{A}$. Then $b=f\left(e_{A}\right)=e_{B}$, implies that $\operatorname{ker} t=\left\{e_{B}\right\}$, i.e., $t$ is injective.

In orrder to show $t$ is surjective, take an element $w N \in D$, where $w \in B * C$, and for $w \neq 1, w$ can be uniquely written as $w=w_{1} \cdots w_{k}$ where all factors are $\neq 1$ and two adjacent factors do not belong to the same group. Then $w N=w_{1} \cdots w_{k} N=$ $w_{1} N \cdots w_{k} N=\varphi\left(w_{1}\right) \cdots \varphi\left(w_{k}\right)$ where $\varphi\left(w_{i}\right)=t\left(w_{i}\right)$ if $w_{i} \in B$ and $\varphi\left(w_{i}\right)=g\left(w_{i}\right)$ if $w_{i} \in C$. If $w_{i} \in C$, then $w_{i}=s\left(a_{i}\right)$ and $g\left(w_{i}\right)=g\left(s\left(a_{i}\right)\right)=g s\left(a_{i}\right)=t f\left(a_{i}\right)$. So $w N=t($ an element of $B) . t$ is surjective. Thus $t$ is an isomorphism, i.e., $t \in S$.

Next let $u: B \rightarrow X$ and $v: C \rightarrow X$ in category $\mathscr{G}$ be such that $u f=v s$.

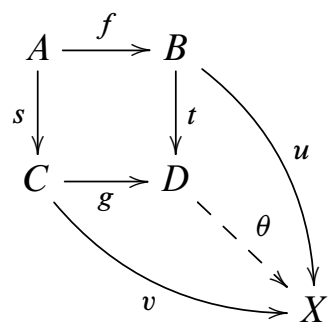

Define $\theta: D \rightarrow X$ by the rule $\theta(w N)=\varphi\left(w_{1}\right) \cdots \varphi\left(w_{k}\right), w=w_{1} \cdots w_{k}$ where $\varphi\left(w_{i}\right)=u\left(w_{i}\right)$ if $w_{i} \in B$ and $\varphi\left(w_{i}\right)=v\left(w_{i}\right)$ if $w_{i} \in C$. We can easily show that $\theta$ is well defined and also a homomorphism. Next we show that the two triangles are commutative. For any $b \in B, \theta t(b)=\theta(b N)=u(b)$ and for any $c \in C$, $\theta g(c)=\theta(c N)=v(c)$. So $\theta t=u$ and $\theta g=v$. This completes the proof of Theorem 3.

Also from the above discussion we conclude the following.

Theorem 4. The category $\mathscr{G}$ is cocomplete.

The proof of the following result is trivial.

Proposition 1. $S$ is saturated.

All the conditions of Theorem 1 ([3], p.32) are satisfied for the category $\mathscr{G}$ and the set of morphisms $S$ of $\mathscr{G}$. So from the Theorem 1.2 ([4], p.63), we have the following result.

Theorem 5. Every object $G$ of the category $\mathscr{G}$ has an Adams completion $G_{S}$ with respect to the set of morphisms $S$. Furthermore, there exists a morphism e: $G \rightarrow G_{S}$ in $S$ which is couniversal with respect to the morphisms in $S:$ given a morphism $s: G \rightarrow H$ in $S$ there exists a unique morphism $t: H \rightarrow G_{S}$ in $S$ such that $t s=e$. 
In other words the following diagram is commutative :

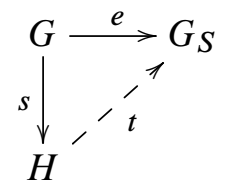

\section{4. $\bar{G}$ AS ADAMS COMPLETION}

We show that $\bar{G}$, a permutation group, as constructed in the proof of Cayley's Theorem for a group $G$, is the Adams completion $G_{S}$ of the group $G$.

Theorem 6. $\bar{G} \cong G_{S}$.

Proof. Consider the following diagram :

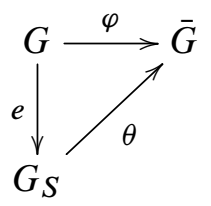

By Theorem 1, there exists a unique morphism $\theta: G_{S} \rightarrow \bar{G}$ in $S$ such that $\theta e=\varphi$. Next consider the following diagram :



By Theorem 5, there exists a unique morphism $\psi: \bar{G} \rightarrow G_{S}$ in $S$ such that $\psi \varphi=e$. Consider the following diagram :

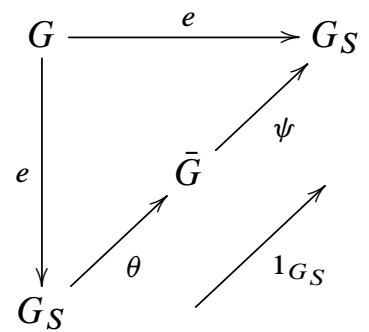

We have $\psi \theta e=\psi \varphi=e$. By the uniqueness condition of the couniversal property of $e$, we conclude $\psi \theta=1_{G_{S}}$. 
Next consider the following diagram :

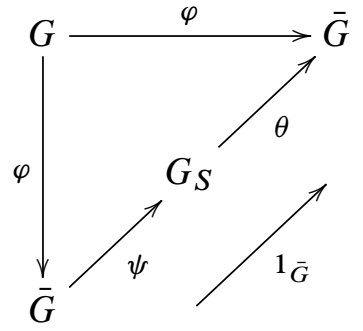

We have $\theta \psi \varphi=\theta e=\varphi$. By the uniqueness condition of the couniversal property of $\varphi$, we conclude $\theta \psi=1_{\bar{G}}$.

Thus $\bar{G} \cong G_{S}$. This completes the proof of Theorem 6 .

\section{ACKNOWLEDGEMENT}

It is a pleasure to thank the unknown reviewer for the comments which resulted in an improved presentation of the paper.

\section{REFERENCES}

[1] J. Adams, Idempotent functors in homotopy theory, Manifolds. Tokyo: Conf. Univ. of Tokyo Press, 1973.

[2] J. Adams, Stable homotopy and generalized homology, ser. Series is books. Chicago and London: The Univ. of Chicago Press, 1974.

[3] A. Deleanu, "Existence of the Adams completions for objects of cocomplete categories," J. Pure and Appl. Alg., vol. 6, pp. 31-39, 1975, doi: 10.1016/0022-4049(75)90010-9.

[4] A. Deleanu, A. Frei, and P. Hilton, "Generalized Adams completions," Cahiers de et Geometrie Differentielle, vol. 15, no. 1, pp. 61-82, 1974.

[5] G. Gallian, Contemporary Abstract Algebra, 7th ed., ser. Series is books. Brooks/Cole Cengage Learning, 2009.

[6] H. Schubert, Categories, ser. Series is books. New York: Springer-Verlag, 1972.

Authors' addresses

\section{Snigdha Bharati Choudhury}

Department of Mathematics, National Institute of Technology Rourkela, Rourkela - 769008 , India E-mail address: snigdha23choudhuryegmail.com

\section{A. Behera}

Department of Mathematics, National Institute of Technology Rourkela, Rourkela - 769 008, India E-mail address: abeheraenitrkl.ac.in 